\title{
Nível de satisfação e perfil dos usuários de um ambulatório escola na cidade de Araguari-MG
}

DOI: $10.5935 / 2447-8539.201900016$

ISABELA MENDES CORREIA; CAROLINE SOARES MENDONÇA ALVES; LARISSA MIRANDA ROCHA; JANYNE DE SÁ ORDERDENGE; INGO YOSHI MATSUBARA GARCIA; WIRLLEY ARANTES DA ROCHA; EURIDES BATISTA FARIA NETTO; JOÃO PEDRO AMORIM LEÃO; AUGUSTO SÉRGIO INÁCIO LEME; HERBERT CRISTIAN DE SOUZA

e-mail: isamcorreia0405@gmail.com

\section{Resumo}

A principal característica dos centros ambulatoriais é o atendimento ao paciente sem que ele tenha sido admitido em ambiente hospitalar. Dessa forma, tem-se como objetivo avaliar o atendimento prestado por ambulatório escola na cidade de Araguari - MG, estabelecendo o perfil socioeconômico dos pacientes assistidos pelos docentes em nível ambulatorial. Serão aplicados questionários aos pacientes, contemplando questões de âmbito socioeconômico e questões que são inerentes ao nível de satisfação do paciente. O levantamento percentual quanto à satisfação dos atendimentos realizados pelo binômio docente e discente, das especialidades mais procuradas e a classificação socioeconômica dos pacientes atendidos são importantes elementos a serem explorados. Este trabalho espera estabelecer a relevância dos resultados encontrados e prover uma perspectiva para ajudar o emprego de ações resolutivas aos problemas levantados pelos pacientes durante a aplicação dos questionários.

Palavras-chave: Satisfação, Ambulatório, Atenção Secundária, Qualidade de atendimento.

\section{Abstract}

The main characteristic of outpatient care centers is to provide healthcare to the patient without the need of in hospital care. The aim of this work is to evaluate the care provided by an outpatient care center in the city Araguari in the state of Minas Gerais, establishing the socioeconomic profile of patients who were assisted in outpatient level. The questionnaires will be submitted to the patients, contemplating questions of socioeconomic scope as well as those that are inherent to patient's level of satisfaction. The percentage survey relating to the satisfaction of the care provided by both teachers and students, the seeking for the medical specialties and the patient socioeconomic profile are important elements to be explored. This work expects to establish the relevance of the found results and provide insight in order to help applying resolute actions to the problems raised by the patients during the questionnaire's submission.

Keywords: Satisfaction, Outpatient care, Secondary Care, Care Quality. 


\section{INTRODUÇÃO}

O Sistema Único de Saúde (SUS) propõe que os serviços de saúde sejam organizados em redes, sendo que cada nível deve oferecer serviços específicos de acordo com sua complexidade, a fim de proporcionar impacto significativo nos níveis de saúde da população (PITANGUEIRA, 2017). O SUS no Brasil é dividido em três áreas básicas de abrangência preconizadas pela Organização Mundial de Saúde (OMS): atenção primária, secundária e terciária.

A primeira engloba serviços como consultas, exames básicos e procedimentos básicos como curativos, seu principal representante são as Unidades Básicas de Saúde. A segunda é representada pelas Unidades de Pronto Atendimento e serviços especializados como os ambulatórios, e a terceira são os hospitais de grande porte (alta complexidade), subsidiados pela esfera privada ou pelo estado. Esses setores aproximam-se em suas práticas de integralidade, bem como na construção de políticas sociais e de saúde para as populações menos favorecidas, em favor da equidade (SANTOS, 2018).

Com relação aos serviços ambulatoriais, é notório a necessidade da participação ativa do paciente no processo de cura e tratamento. Em meio a isso o estímulo ao autocuidado, embasado no processo de educação em saúde, torna-se, portanto, o propósito maior do seguimento ambulatorial no intuito de capacitar o cliente (PESSOA, 2017). Por esse viés, tornase imprescindível a satisfação do paciente a nível ambulatorial a fim de que ele se sinta motivado a seguir orientações e planos de cuidados.

A principal característica dos centros ambulatoriais é o atendimento ao paciente sem que ele tenha sido admitido em ambiente hospitalar. 0 aprendizado neste cenário, que é centrado no paciente, tem sido considerado um componente essencial da educação médica, permitindo com que alunos e preceptores atuem no processo de atenção ao paciente. Independentemente da metodologia educacional escolhida para ser utilizada no ambiente ambulatorial, pacientes esperam ser diagnosticados e tratados adequadamente (BRAGA, 2015).

Estudos científicos realizados em torno dessa temática visam buscar falhas na saúde básica e transplantar dados coletados na melhoria coletiva. A intenção real de se obter o nível de satisfação dos usuários é, principalmente, a de transformar essas informações em medidas que permitirão o aperfeiçoamento dos serviços. Além disso, traçar o perfil dos pacientes locais contribui significativamente para essa análise. A satisfação dos usuários é uma meta a ser alcançada pelos serviços e dessa maneira deve ser pesquisada, visando o aperfeiçoamento do atendimento (BASTOS, 2013).
Um estudo realizado no hospital escola da cidade de São Carlos aponta que os pacientes ficaram na maioria de muito satisfeitos $(46,2 \%)$ a satisfeitos $(50,0 \%)$ com a agilidade no atendimento do hospital. Além disso em relação ao ambiente físico hospitalar, os usuários de todos os serviços consideraram muito boa a limpeza $(72,3 \%)$, muito bom o conforto $(53,8 \%)$ e a sinalização $(73,1 \%)$ do hospital. Com relação ao atendimento da equipe geral $96,4 \%$ dos usuários ficaram muito satisfeitos a satisfeitos (RICCI, 2011).

A fim de comparação, em um estudo de pesquisa de satisfação de atendimento ambulatorial realizado na Alemanha homens (em relação a mulheres) e indivíduos idosos possuem o índice de satisfação ligeiramente mais elevado quando se considera 4 elementos primordiais na prática médica: organização, informação, interação e competência profissional (AURAS, 2016).

Por tudo isso, ao se tratar de saúde pública as problemáticas e as pesquisas em torno da satisfação e perfil dos pacientes a unidades e centros de saúde devem ser investigadas a fim de buscar sempre o bem coletivo. A partir da década de 70, essas pesquisas ganharam notoriedade, principalmente em países desenvolvidos, devido ao recrudescimento do consumo e à exigência da qualidade pelos consumidores (BASTOS, 2013).

\section{METODOLOGIA}

Trata-se de um estudo observacional de corte transversal, de abordagem quantitativa, onde os participantes responderão a um questionário virtual elaborado pelos próprios pesquisadores, visando sintetizar as principais características que delinearão o perfil do paciente e sua avaliação mediante o serviço prestado. Os questionários serão aplicados em diferentes horários e dias da semana, entre 8:00 e 18:00 horas, de segunda a sexta feira, de fevereiro a junho de 2019. Também serão analisados os dados do sistema de cadastro do ambulatório, para o levantamento da frequência e especialidades procuradas por seus assistidos.

A população estimada de assistidos pelo ambulatório é de aproximadamente 1.700 indivíduos por mês, divididos em 24 especialidades. Para o cálculo do tamanho da amostra, utilizaram-se um nível de confiança de 95\%, uma proporção esperada para o evento de $50 \%$ e uma precisão de $5 \%$.

Dessa forma, encontrou-se um n mínimo necessário de aproximadamente 370 pacientes, ou seja aproximadamente $4,34 \%$ dos pacientes de cada especialidade. Os pacientes que participarão da pesquisa serão selecionados por meio da técnica de 
amostragem aleatória simples e estratificada por especialidade.

Os dados serão tabulados e avaliados por meio de uma análise descritiva de valores absolutos e relativos. Caso pertinente também será feito o tratamento inferencial dos mesmos, por meio de testes de hipóteses específicos tais como: qui-quadrado, teste $G$, dentre outros, com o auxílio dos softwares Excel e Bioestat.

Para seleção, será adotado como critérios de inclusão: serem pacientes alfabetizados de ambos os sexos; com idade igual ou superior a 18 anos e apresentarem aptidão física e mental para responder o questionário. 0 risco possível seria a exposição do participante, no entanto os pesquisadores se comprometem com o sigilo das informações prestadas, bem como com a não identificação deles.

O benefício desta pesquisa é de grande valia pois, os achados podem servir como norteadores e até mesmo como ferramentas de gestão, visando a excelência na qualidade dos serviços prestados pelo ambulatório à comunidade.

Para garantir os direitos e integridade ética, torna-se necessário o Termo de Consentimento Livre e Esclarecido (TCLE), o Termo de Consentimento da Instituição e a aprovação pelo Comitê de Ética e Pesquisa (CEP).

Foi utilizado o Net Promoter Score na análise da "Qualidade de atendimento pelo aluno", "Qualidade de atendimento pelo médico", "Tempo de espera". Essa análise permite identificar pontos onde a melhora na percepção de qualidade do usuário dos serviços deve ser reforçada e adequada.

Em um NPS de $-100 \%$ a 0\%, tem-se a classificação "Crítica", que indica necessidade de intervenção urgente. Quando o NPS fica entre $1 \%$ e $30 \%$, é um indicativo de "Aperfeiçoamento" de caráter importante. Na faixa de $31 \%$ a $70 \%$, o NPS indica um serviço de "Qualidade", porém com pontos de melhoria identificáveis. Finalmente, de $71 \%$ a $100 \%$ é indicado um serviço de "Excelência".

\section{RESULTADOS E DISCUSSÕES}

Foram entrevistados 370 pacientes no ambulatório de educação em saúde. Desses, 295 eram do sexo feminino e 75 do sexo masculino. Essa informação nos mostra uma maioria expressiva de mulheres.

Conhecendo tal fato, medidas podem ser tomadas, como a criação de programas para a saúde da mulher, tratando-se do grupo predominante. Ou ainda, programas de incentivem a saúde do homem, como forma de atrair esse outro grupo.
Em relação a idade, o grupo etário mais prevalente foi de 46 a 60 anos. Na escolaridade, 119 pessoas apresentavam ensino fundamental incompleto. Entender o perfil de escolaridade é um ponto extremamente importante, e que pode ter grande impacto na adesão ao tratamento.

Estudo transversal exploratório realizado em unidade de saúde da família de Londrina, Paraná, em 2007, mostrou associação positiva entre escolaridade e adesão à tratamento não farmacológico. Em relação a renda, 178 tinham até 3 salários mínimos, e 170 tinham até 1 . A renda pode ser um fator comprometedor da adesão à terapia farmacológica. Estudo descritivo, realizado em hospital universitário, em 2009, mostrou que quanto maior o grau de instrução e capacidade econômica, menor a incidência de doenças.

Essa relação apresenta vários aspectos e pode direcionar a conduta e as orientações no atendimento e infere diretamente em como os pacientes avaliam a sua melhora em seu estado de saúde após o início do tratamento ambulatorial. Essa avaliação foi consideravelmente alta, com valor de $43 \%$ para aqueles que consideravam "muita melhora" e 8\% para aqueles com "completa" melhora. No que tange aos aspectos negativos, 14\% referiu "pouca melhora" e 13\% "nenhuma melhora". Dentro dos valores avaliados, temse ainda $21 \%$ que relata uma melhora da saúde em nível médio e $1 \%$ não quis responder este quesito (Gráfico 1).

Gráfico 1 - Melhoria na saúde os pacientes

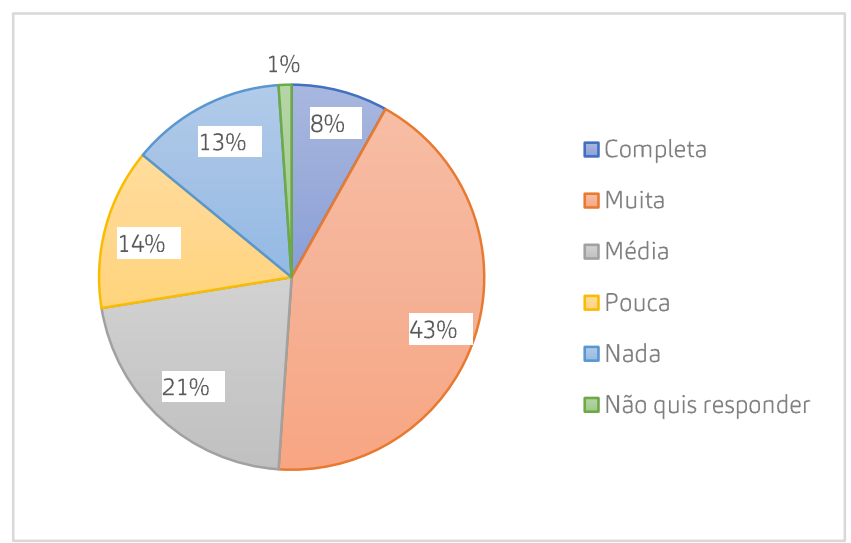

Outro aspecto pertinente é o número de consultas. Dessa forma, essa avaliação respeitou o critério propostos em que pessoas com menos de uma consulta ambulatorial não respondeu ao questionário e, com isso, qualquer paciente entrevistado apresentou no mínimo de uma consulta prévia, resultando assim em um número superior de consultas ao número de pacientes entrevistados.

Sendo assim, foram abordadas 20 especialidades em 544 consultas prévias, entre as quais se destacam a 
Ginecologia e Obstetrícia com 169 consultas ou 31\% do número total de consultas o que pode estar associado a proporção quatro vezes maior de pacientes entrevistados do sexo feminino em relação ao número de pacientes do sexo masculino, e em segundo lugar a Clínica Geral com 157 consultas prévias/ ou $28 \%$.

Endocrinologia e Cardiologia aparecem consideravelmente somando as duas especialidades 87 consultas ou $16 \%$ do número total de consultas. Em números bem menores, com número de consultas prévias menor ou igual a 20, aparecem em ordem decrescente: reumatologia, Nutrição, Pneumologia, Urologia, Dermatologia, Otorrinolaringologia, Gastrologia, Neurologia, Mastologia, Oftalmologia, Angiologia, Nefrologia, Cirurgia, Tabagismo, Geriatria e Infectologia, o que somado resulta em 140 consultas ou 25\% do número total de consultas.

Além disso, foram analisados os dados referentes ao entendimento do paciente quanto a sua doença e tratamento. Assim, afirmaram entender bem 41\% (151), entender completamente38\% (139) e apenas 3\% (13) não conhecem o tratamento. Não obstante, no que se refere à qualidade do atendimento do médico, avaliaram como excelente 67\%(246) e bom 31\%(116).

Sendo assim, sugere-se que, partindo de um excelente atendimento médico, centrado no paciente, levando em conta aspectos éticos e morais, a compreensão dos pacientes quanto à sua doença e tratamento faz-se de forma eficaz e de grande valia. De acordo com Rilva Lopes et al, 2012 inúmeras vezes as orientações e explicações sobre a doença e o tratamento do paciente não é exposta de forma pertinente por parte do médico, que acaba por dificultar o processo terapêutico.

Ao avaliar a qualidade do atendimento pelos médicos e pelos alunos por meio do Net Promoter Score, percebese que ambos serviços estão em níveis de qualidade muito semelhantes, sendo que "Qualidade de atendimento pelos alunos" tem um NPS de $62,43 \%$, enquanto a "Qualidade de atendimento pelos médicos" tem um NPS de 64,32\%. Ambos se encontram na classificação de "Qualidade" estabelecida pelo método NPS. Percebe-se que ambos serviços têm qualidade avaliada positivamente, porém com pontos de possíveis melhorias.

Ao tratar-se atendimentos ambulatoriais, é fundamental o entendimento do paciente no que tange ao tempo de espera antes da consulta médica. Dessa maneira, este foi classificado como "Aperfeiçoamento" com um NPS de 5,9\%. Esse dado revela um ponto importante para melhoria na percepção de qualidade do usuário do ambulatório, apesar de grande parte dos usuários iniciarem suas consultas em horário agendado ou com atraso de até 30 minutos. De acordo com Wang. W (2019), em um estudo realizado na parte rural da China, o tempo de espera não foi um determinante forte para a qualidade do atendimento percebida pelos usuários.

Algumas melhorias estruturais foram apontadas pelos entrevistados durante estudo. Através dos questionários aplicados aos pacientes, aferimos que as melhorias sugeridas por eles no Ambulatório Romes Nader são em relação à pontualidade e à disponibilidade de datas para consultas. Sendo pontuadas por 32 e 107 pessoas, respectivamente.

De acordo com Moimaz et al. $(2010$, p. 8) foi afirmado que há uma expressiva demora do dia que é feito o agendamento tanto da consulta, quanto do exame, até o atendimento por 54,5\% da população. Partindo do pressuposto de que é um dado relativo à percepção pessoal, sendo que o tempo de espera ao qual os pacientes são submetidos pode ser desde dias à anos.

Nesse estudo 73\% dos entrevistados classificaram o respeito e cordialidade com o paciente como Excelente, $26 \%$, como Bom, $4 \%$, como Médio, e menos de $1 \%$ como ruim e péssimo. Sendo assim vale ressaltar que o aspecto humanitário de cordialidade está sendo bem empregado no Ambulatório de especialidades, o que é um ponto positivo sendo que diversos autores vem enfatizando a satisfação dos pacientes quanto ao trato humanitário e a relação-médico paciente (FRANCO et. al.1998), de modo que esta seja um indicador de melhora na qualidade do serviço de saúde ( HERCOS et. al. 2006).

A avaliação estrutural do ambulatório estudado demonstrou ser de qualidade excelente visto que no presente estudo dos 370 entrevistados, 299 classificaram como excelente, 70 como bom, 1 como médio, nenhum como ruim e nenhum como péssimo.

Deste modo vale ressaltar a importância de um ambiente propício aos cuidados da saúde, visto que condições físicas precárias têm impacto sobre a ação profissional e o conforto dos paciente, podendo inclusive influenciar negativamente a história da doença (GOMES et al., 2011)

\section{CONCLUSÕES}

De acordo com os dados obtidos, observa se que, em linhas gerais, os serviços prestados no ambulatório estão sendo satisfatórios para a população atendida.

Análise de variáveis como o bom entendimento da doença e o excelente respeito e empatia refletem a classificação excelente de qualidade do atendimento tanto pelos médicos, quanto pelos alunos evidenciada pela maioria dos entrevistados.

Além disso, deve se destacar a questão da pontualidade e da disponibilidade para agendamento de consultas, como pontos para serem aprimorados 
estrategicamente pela gestão do ambulatório a fim de garantir um maior conforto para os pacientes.

Nessa perspectiva, portanto, fica clara a importância de realizar uma avaliação dos serviços prestados pelo ambulatório tendo como ponto de partida a visão do paciente. Assim, é possível analisar por meio de um referencial externo quais pontos devem ser melhorados e quais devem ser mantidos para que o paciente tenha, sobretudo, uma experiência satisfatória ao comparecer à consulta.

\section{REFERÊNCIAS}

AURAS, S. et al. Patient satisfaction with ambulatory care in Germany: effects of patient-and medical practice-related factors. International Journal for Quality in Health Care, v. 28, n. 6, p. 808-815, 2016.

BASTOS, G. A. N.; FASOLO, L. R. Fatores que influenciam a satisfação do paciente ambulatorial em uma população de baixa renda: um estudo de base populacional. Revista Brasileira de Epidemiologia, v. 16, п. 1, p. 114-124, 2013.

BRAGA, L. P. M. Supervisão docente mínima e indireta: percepções dos pacientes em diferentes cenários de prática. 2015. 76 f. Tese (Doutorado) universidade Federal do Mato Grosso do Sul, 2015.

DE SOUSA-MUÑOZ, R. L. et al. Informação do paciente sobre sua doença e terapêutica em projeto de extensão universitária. Revista Brasileira em Promoção da Saúde, v. 24, n. 3, p. 258-265, 2012.

FRANCO, S. C.; CAMPOS, G. W. de S. Avaliação da qualidade de atendimento ambulatorial em pediatria em um hospital universitário. Cadernos de Saúde Pública, v. 14, p. 61-70, 1998.

GIROTTO, E. et al. Adesão ao tratamento farmacológico e não farmacológico e fatores associados na atenção primária da hipertensão arterial. Ciência \& Saúde Coletiva, v. 18, p. 1763-1772, 2013.

GOMES, I. P.; COLLET, N.; DOS REIS, P. E. D. Ambulatório de quimioterapia pediátrica: a experiência no aquário carioca. Texto \& Contexto Enfermagem, v. 20, п. 3, p. 385-391, 2011.

HERCOS, B. V. S.; BEREZOVSKY, A. Qualidade do serviço oftalmológico prestado aos pacientes ambulatoriais do Sistema Único de SaúdeSUS. Arquivo Brasileiro de Oftalmologia, v. 69, п. 2, p. 213-9, 2006
MOIMAZ, S. A. S., et al. Satisfação e percepção do usuário do SUS sobre o serviço público de saúde. Physis: Revista de Saúde Coletiva, 20, 14191440, 2010

PESSOA, Vera Lúcia Mendes de Paula et al. Assistência de enfermagem ambulatorial: percepção de transplantados cardíacos sobre a consulta de enfermagem ambulatorial. Rev. pesqui. cuid. fundam.(Online), v. 9, n. 4, p. 984-989, 2017.

PITANGUEIRA, Cássia Menaia FC; PAIVA, Carlos Henrique Assunção. A atenção básica e a rede de saúde de Juazeiro, Bahia (2001-2012): uma análise neoinstitucionalista. Revista Baiana Saúde Pública, v. 41, п. 1, p. 41. п1. 2075, 2017.

RICCI, Natalia Aquaroni et al. O hospital-escola de São Carlos: análise do funcionamento por meio da satisfação dos usuários. Ciência \& Saúde Coletiva, v. 16, p. 1125-1134, 2011.

SANTOS, Carla Targino Bruno dos et al. A integralidade no Brasil e na Venezuela: similaridades e complementaridades. Ciencia \& Saúde Coletiva, v. 23, p. 1233-1240, 2018. 